Georgian Mathematical Journal

Volume 13 (2006), Number 3, 585-598

\title{
OSCILLATIONS OF HIGHER ORDER FUNCTIONAL DIFFERENTIAL EQUATIONS WITH IMPULSES
}

\author{
CHAOLONG ZHANG AND WEIZHEN FENG
}

\begin{abstract}
A kind of higher order sub-and super-linear FDE with impulses is studied in this paper. Several criteria on the oscillations of solutions are given. In particular, in the case where the coefficients of equations are positive and continuous functions, we find some suitable impulse functions such that all solutions of the equation are oscillatory under the impulse control.
\end{abstract}

2000 Mathematics Subject Classification: 34A37.

Key words and phrases: Higher order, FDE, impulses, oscillation.

\section{INTRODUCTION}

Recent years have seen on increasing number of papers dealing with the oscillatory behavior of ODE(FDE) with impulses. There are some good results on the oscillation of first order ODE with impulses [1]-[5]. The oscillation of second order ODE with impulses is studied in [6]-[8], and the oscillation of second order FDE in [9]-[11]. Some results on the oscillation of higher order ODE are obtained in [12], [13]. However papers, where the oscillation of higher order FDE is investigated, are very rare.

In this paper, we mainly study a kind of higher order sub- and super-linear FDE with impulses under conditions $(A)$ and $(B)$. We can always find some suitable impulse functions such that all solutions of the equation can become oscillatory under the impulse control. We believe that the oscillation under the impulse control is significant both in the theory and in applications.

\section{Main Results}

We consider the system

$$
\left\{\begin{array}{l}
x^{(2 n)}(t)+p(t)|x(t-\tau)|^{r} \operatorname{sgn}(x(t-\tau))=0, \quad t \geq t_{0}, \quad t \neq t_{k}, \\
x^{(i)}\left(t_{k}^{+}\right)=a_{k}^{(i)} x^{(i)}\left(t_{k}\right), \quad i=0,1, \ldots, 2 n-1, \quad k=1,2, \ldots \\
x^{(i)}\left(t_{0}^{+}\right)=x_{0}^{(i)}, \quad i=0,1, \ldots, 2 n-1, \\
x(t)=\phi(t), \quad t_{0}-\tau \leq t \leq t_{0}
\end{array}\right.
$$

where

$$
\begin{aligned}
x^{(i)}\left(t_{k}\right) & =\lim _{h \rightarrow 0^{-}} \frac{x^{(i-1)}\left(t_{k}+h\right)-x^{(i-1)}\left(t_{k}\right)}{h}, \\
x^{(i)}\left(t_{k}^{+}\right) & =\lim _{h \rightarrow 0^{+}} \frac{x^{(i-1)}\left(t_{k}+h\right)-x^{(i-1)}\left(t_{k}^{+}\right)}{h},
\end{aligned}
$$

ISSN 1072-947X / \$8.00 / (C) Heldermann Verlag www.heldermann.de 
$\phi:\left[t_{0}-\tau, t_{0}\right] \rightarrow R$ has at most a finite number of discontinuous points of first kind and is left continuous at these points, $0 \leq t_{0}<t_{1}<\cdots<t_{k}<\cdots$, $\lim _{k \rightarrow \infty} t_{k}=+\infty, x^{(0)}(t)=x(t), n$ is a natural number. Here we always assume that the following conditions hold:

(A) $a_{k}^{(i)}>0, i=0,1, \ldots, 2 n-1, \tau \geq 0, r>0, t_{k+1}-t_{k}>\tau, p(t)$ is nonnegative and continuous on $\left[t_{0},+\infty\right)$, and $p(t)$ is not always equal to 0 in $[t,+\infty)$ for $t \geq t_{0}$;

$$
\begin{aligned}
& \left(t_{1}-t_{0}\right)+\frac{a_{1}^{(i)}}{a_{1}^{(i-1)}}\left(t_{2}-t_{1}\right)+\frac{a_{1}^{(i)} a_{2}^{(i)}}{a_{1}^{(i-1)} a_{2}^{(i-1)}}\left(t_{3}-t_{2}\right) \\
& \quad+\cdots+\frac{a_{1}^{(i)} a_{2}^{(i)} \cdots a_{m}^{(i)}}{a_{1}^{(i-1)} a_{2}^{(i-1)} \cdots a_{m}^{(i-1)}}\left(t_{m+1}-t_{m}\right)+\cdots=+\infty
\end{aligned}
$$

Definition 1. A function $x:\left[t_{0}-\tau,+\infty\right) \rightarrow R$ is said to be a solution of (1) on $\left[t_{0}-\tau,+\infty\right)$ starting from $\left(t_{0}, \phi, x_{0}^{(0)}, x_{0}^{(1)}, \ldots, x_{0}^{(2 n-1)}\right)$ if

(i) $x^{(i)}(t)$ is continuous on $\left[t_{0},+\infty\right) \backslash\left\{t_{k}, k \in N\right\}, i=0,1, \ldots, 2 n-1$;

(ii) $x(t)=\phi(t), t \in\left[t_{0}-\tau, t_{0}\right], x^{(i)}\left(t_{0}^{+}\right)=x_{0}^{(i)}, i=0,1, \ldots, 2 n-1$;

(iii) $x(t)$ satisfies the first equality of (1) on $\left[t_{0},+\infty\right) \backslash\left\{t_{k}, k \in N\right\}$;

(iv) $x^{(i)}(t)$ has two-side limits and is left continuous at the points $t_{k}, k=$ $1,2, \ldots, x^{(i)}\left(t_{k}\right)$ satisfies the second equality of $(1), i=0,1,2, \ldots, 2 n-1$.

Remark 1. Let $x_{0}(t)=x(t), x_{1}(t)=x^{\prime}(t), \ldots, x_{2 n-1}(t)=x^{(2 n-1)}(t)$. Then equation (1) can be changed into

$$
\left\{\begin{array}{l}
x_{0}^{\prime}(t)=x_{1}(t) \\
x^{\prime}{ }_{1}(t)=x_{2}(t) \\
\ldots \ldots \ldots \ldots \\
x^{\prime}{ }_{2 n-2}(t)=x_{2 n-1}(t), \quad t \geq t_{0}, \quad t \neq t_{k}, \\
x^{\prime}{ }_{2 n-1}(t)=-p(t)\left|x_{0}(t-\tau)\right|^{r} \operatorname{sgn} x_{0}(t-\tau), \\
x_{i}\left(t_{k}^{+}\right)=a_{k}^{(i)} x_{i}\left(t_{k}\right), \quad i=0,1, \ldots, 2 n-1, \quad k=1,2 \ldots \\
x_{0}(t)=\phi(t), \quad t_{0}-\tau \leq t \leq t_{0}, \\
x^{(i)}\left(t_{0}^{+}\right)=x_{0}^{(i)} .
\end{array}\right.
$$

The global existence and uniqueness of solution of (1) can be found in [14]-[15]. In the following, we always assume that solutions of (1) exist on $\left[t_{0},+\infty\right)$.

Definition 2. A solution of (1) is said to be non-oscillatory if it is eventually positive or eventually negative. Otherwise, this solution is said to be oscillatory.

Lemma 1. Let $x(t)$ be a solution of $(1)$, and conditions $(A),(B)$ be satisfied. Suppose that there exist an $i \in\{1,2, \ldots, 2 n-1\}$ and some $T \geq t_{0}$ such that $x^{(i)}(t)>0(<0), x^{(i+1)}(t) \geq 0(\leq 0)$ for $t \geq T$. Then there exists some $T_{1} \geq T$ such that $x^{(i-1)}(t)>0(<0)$ for $t \geq T_{1}$. 
Proof. Without loss of generality, suppose that $T=t_{1}, x^{(i)}(t)>0, x^{(i+1)}(t) \geq 0$ for $t \in\left(t_{k}, t_{k+1}\right](k=1,2, \ldots)$. Hence $x^{(i)}(t)>0$ is monotonically nondecreasing in $\left(t_{k}, t_{k+1}\right]$. For $t \in\left(t_{1}, t_{2}\right]$, we have

$$
x^{(i)}(t) \geq x^{(i)}\left(t_{1}^{+}\right)
$$

Integrating the above inequality, we have

$$
x^{(i-1)}\left(t_{2}\right) \geq x^{(i-1)}\left(t_{1}^{+}\right)+x^{(i)}\left(t_{1}^{+}\right)\left(t_{2}-t_{1}\right) .
$$

Similarly to (3),

$$
x^{(i-1)}\left(t_{3}\right) \geq x^{(i-1)}\left(t_{2}^{+}\right)+x^{(i)}\left(t_{2}^{+}\right)\left(t_{3}-t_{2}\right) .
$$

By $x^{(i)}\left(t_{2}\right) \geq x^{(i)}\left(t_{1}^{+}\right)$and $(3),(4)$, we have

$$
\begin{aligned}
x^{(i-1)}\left(t_{3}\right) & \geq x^{(i-1)}\left(t_{2}^{+}\right)+x^{(i)}\left(t_{2}^{+}\right)\left(t_{3}-t_{2}\right) \\
& =a_{2}^{(i-1)} x^{(i-1)}\left(t_{2}\right)+a_{2}^{(i)} x^{(i)}\left(t_{2}\right)\left(t_{3}-t_{2}\right) \\
& \geq a_{2}^{(i-1)}\left[x^{(i-1)}\left(t_{1}^{+}\right)+x^{(i)}\left(t_{1}^{+}\right)\left(t_{2}-t_{1}\right)\right]+a_{2}^{(i)} x^{(i)}\left(t_{2}\right)\left(t_{3}-t_{2}\right) \\
& \geq a_{2}^{(i-1)}\left[x^{(i-1)}\left(t_{1}^{+}\right)+x^{(i)}\left(t_{1}^{+}\right)\left(t_{2}-t_{1}\right)+\frac{a_{2}^{(i)}}{a_{2}^{(i-1)}} x^{(i)}\left(t_{1}^{+}\right)\left(t_{3}-t_{2}\right)\right] .
\end{aligned}
$$

Applying mathematical induction for any natural number $m$ we have

$$
\begin{aligned}
x^{(i-1)}\left(t_{m}\right) \geq & a_{m-1}^{(i-1)} \cdots a_{3}^{(i-1)} a_{2}^{(i-1)}\left\{x^{(i-1)}\left(t_{1}^{+}\right)+x^{(i)}\left(t_{1}^{+}\right)\left[\left(t_{2}-t_{1}\right)\right.\right. \\
& \left.\left.+\frac{a_{2}^{(i)}}{a_{2}^{(i-1)}}\left(t_{3}-t_{2}\right)+\cdots+\frac{a_{2}^{(i)} a_{3}^{(i)} \cdots a_{m-1}^{(i)}}{a_{2}^{(i-1)} a_{3}^{(i-1)} \cdots a_{m-1}^{(i-1)}}\left(t_{m}-t_{m-1}\right)\right]\right\} .
\end{aligned}
$$

By condition (B) and $a_{k}^{(i)}>0$, for all sufficiently large $m$, we have $x^{(i-1)}\left(t_{m}\right)>0$. That is, there exists a natural number $N$ such that $t_{N} \geq T$ and for $m \geq N$, we have $x^{(i-1)}\left(t_{m}\right)>0$. Since $x^{(i)}(t)>0$, we have $x^{(i-1)}(t)>x^{(i-1)}\left(t_{k}\right)>0$ for $t \in\left(t_{k}, t_{k+1}\right]$ where $k \geq N$. Hence for $t \geq t_{N}$, we have $x^{(i-1)}(t)>0$. The proof of Lemma 1 is completed.

Lemma 2. Let $x(t)$ be a solution of $(1)$ and conditions $(A),(B)$ be satisfied. Suppose that there exist an $i \in\{1,2, \ldots, 2 n\}$ and some $T \geq t_{0}$ such that $x(t)>$ $0, x^{(i)}(t) \leq 0$ for $t \geq T, x^{(i)}(s)$ is not always equal to 0 in $[t,+\infty)(t \geq T)$. Then $x^{(i-1)}(t)>0$ for all sufficiently large $t$.

Proof. Let $T=t_{0}$. We claim that $x^{(i-1)}\left(t_{k}\right)>0$ for any $t_{k} \geq T$.

If this is not true, then there exists some $t_{j} \geq T$ such that $x^{(i-1)}\left(t_{j}\right) \leq 0$. Since $x^{(i)}(t) \leq 0, x^{(i-1)}(t)$ is non-increasing in $\left(t_{k}, t_{k+1}\right]$ for $k \geq j$ and $x^{(i)}(s)$ is not always equal to 0 in $[t,+\infty)$, there exists some $t_{l} \geq t_{j}$ such that $x^{(i)}(t)$ is not always equal to 0 in $\left(t_{l}, t_{l+1}\right]$. Without loss of generality, we can assume that $l=j$. So we have

$$
x^{(i-1)}\left(t_{j+1}\right)<x^{(i-1)}\left(t_{j}^{+}\right)=a_{j}^{(i-1)} x^{(i-1)}\left(t_{j}\right) \leq 0 .
$$


For $t \in\left(t_{j+1}, t_{j+2}\right]$ we have

$$
x^{(i-1)}\left(t_{j+2}\right) \leq x^{(i-1)}\left(t_{j+1}^{+}\right)=a_{j+1}^{(i-1)} x^{(i-1)}\left(t_{j+1}\right)<0 .
$$

By induction, we have $x^{(i-1)}(t)<0, t \in\left(t_{j+m}, t_{j+m+1}\right]$ for all sufficiently large $m$. Thus we have $x^{(i-1)}(t)<0, x^{(i)}(t) \leq 0, t \in\left(t_{j+1},+\infty\right)$. By Lemma 1 , for all sufficiently large $t$ we have $x^{(i-2)}(t)<0$. Applying Lemma 1 repeatedly, for all sufficiently large $t$ we have $x(t)<0$. This is a contradiction to $x(t)>0(t \geq T)$ ! Hence we have $x^{(i-1)}\left(t_{k}\right)>0$ for any $t_{k}$. So we have $x^{(i-1)}(t)>0$ for all sufficiently large $t$. The proof of Lemma 2 is completed.

Lemma 3. Let $x(t)$ be a solution of $(1)$ and conditions $(A),(B)$ be satisfied. Suppose there exist some $T \geq t_{0}$ such that $x(t)>0$ for $t \geq T$. Then there exists some $T^{\prime} \geq T$ and $l \in\{1,3, \ldots, 2 n-1\}$ such that for $t \geq T^{\prime}$,

$$
\left\{\begin{array}{l}
x^{(i)}(t)>0, \quad i=0,1, \ldots, l \\
(-1)^{i-1} x^{(i)}(t)>0, \quad i=l+1, \ldots, 2 n-1, \\
x^{(2 n)}(t) \leq 0
\end{array}\right.
$$

Proof. Let $T=t_{0}$. Since $x(t)>0\left(t \geq t_{0}\right)$, by $(1)$ and that $p(t)$ is nonnegative and is not always equal to 0 on any $(t,+\infty)$, we have

$$
x^{(2 n)}(t)=-p(t)[x(t-\tau)]^{r} \leq 0,
$$

and $x^{(2 n)}(s)$ is not always equal to 0 in $(t,+\infty)$ for $t \geq t_{0}$. By Lemma 2, we have $x^{(2 n-1)}(t)>0$ for sufficiently large $t$. Without loss of generality, let $x^{(2 n-1)}(t)>0$ for $t \geq t_{0}$. So $x^{(2 n-2)}(t)$ is nondecreasing on $\left(t_{k}, t_{k+1}\right]$. If for any $t_{k}, x^{(2 n-2)}\left(t_{k}\right)<0$, then $x^{(2 n-2)}(t)<0\left(t \geq t_{0}\right)$. If there exists some $t_{j}$ such that $x^{(2 n-2)}\left(t_{j}\right) \geq 0$, by $x^{(2 n-2)}(t)$ is nondecreasing on $\left(t_{k}, t_{k+1}\right]$ and $a_{k}^{(2 n-2)}>0$, we get $x^{(2 n-2)}(t)>0$ for $t>t_{j}$. So there exists some $T_{1} \geq T$ such that one of the following statements holds:

$$
\begin{aligned}
& \left(A_{1}\right) \quad x^{(2 n-1)}(t)>0, \quad x^{(2 n-2)}(t)>0, \quad t \geq T_{1} ; \\
& \left(B_{1}\right) \quad x^{(2 n-1)}(t)>0, \quad x^{(2 n-2)}(t)<0, \quad t \geq T_{1} .
\end{aligned}
$$

When $\left(A_{1}\right)$ holds, by Lemma 1 , we have $x^{(2 n-3)}(t)>0$ for all sufficiently large $t$. Applying Lemma 1 repeatedly, for all sufficiently large $t$, we have $x^{(2 n-1)}(t)>0$, $x^{(2 n-2)}(t)>0, \ldots, x^{\prime}(t)>0, x(t)>0$, and (6) holds with $l=2 n-1$.

When $\left(B_{1}\right)$ holds, by Lemma 2 , we have $x^{(2 n-3)}(t)>0$ for all sufficiently large $t$. By deducing further, there exists some $T_{2} \geq T_{1}$ such that one of the following statements holds:

$$
\begin{array}{ll}
\left(A_{2}\right) & x^{(2 n-3)}(t)>0, \quad x^{(2 n-4)}(t)>0, \quad t \geq T_{2} ; \\
\left(B_{2}\right) & x^{(2 n-3)}(t)>0, \quad x^{(2 n-4)}(t)<0, \quad t \geq T_{2} .
\end{array}
$$

Repeating the above reasoning, we can get that there exists some $T^{\prime} \geq T$ and 
$l \in\{1,3, \ldots, 2 n-3\}$ such that for $t \geq T^{\prime}$,

$$
\left\{\begin{array}{l}
x^{(i)}(t)>0, \quad i=0,1, \ldots, l \\
(-1)^{i-1} x^{(i)}(t)>0, \quad i=l+1, l+2, \ldots, 2 n-1, \\
x^{(2 n)}(t) \leq 0
\end{array}\right.
$$

The proof of Lemma 3 is completed.

Remark 2. If $x(t)$ is an eventually negative solution of (1), we obtain results similar to Lemmas 2 and 3.

Theorem 1. If conditions $(A),(B)$ hold, $a_{k}^{(0)} \geq 1$ for $k=1,2, \ldots$ and

$$
\begin{aligned}
\int_{t_{0}}^{t_{1}} p(t) d t+\frac{1}{a_{1}^{(2 n-1)}} \int_{t_{1}}^{t_{2}} p(t) d t+\frac{1}{a_{1}^{(2 n-1)} a_{2}^{(2 n-1)}} \int_{t_{2}}^{t_{3}} p(t) d t \\
+\cdots+\frac{1}{a_{1}^{(2 n-1)} a_{2}^{(2 n-1)} \cdots a_{m}^{(2 n-1)}} \int_{t_{m}}^{t_{m+1}} p(t) d t+\cdots=+\infty,
\end{aligned}
$$

then every solution of (1) is oscillatory.

Proof. Let $x(t)$ be a non-oscillatory solution of (1). Without loss of generality, let $x(t)>0\left(t \geq t_{0}\right)$. By Lemma 3 and (1), there exists $T^{\prime} \geq t_{0}$ such that for $t \geq T^{\prime}$ we have

$$
x^{(2 n)}(t) \leq 0, \quad x^{(2 n-1)}(t)>0, \quad x^{\prime}(t)>0, \quad x(t)>0 .
$$

Since $a_{k}^{(0)} \geq 1(k=1,2, \ldots)$, there exists some natural number $l$ such that $x(t)$ is nondecreasing in $\left[t_{l},+\infty\right), x\left(t_{l}^{+}\right) \leq x\left(t_{l+1}\right) \leq x\left(t_{l+1}^{+}\right) \leq x\left(t_{l+2}\right) \leq x\left(t_{l+2}^{+}\right) \leq \cdots$. Let $j$ be some natural number such that $\left[t_{j}-\tau,+\infty\right) \subset\left[t_{l},+\infty\right)$. Then (1) yields

$$
x^{(2 n)}(t)=-p(t) x^{r}(t-\tau), \quad t>t_{j}, \quad t \neq t_{k} .
$$

Integrating (9) from $t_{j}$ to $t_{j+1}$, we have

$$
x^{(2 n-1)}\left(t_{j+1}\right)-x^{(2 n-1)}\left(t_{j}^{+}\right)=-\int_{t_{j}}^{t_{j+1}} p(t) x^{r}(t-\tau) d t .
$$

By (10) and that $x(t)$ is increasing, we have

$$
\begin{aligned}
x^{(2 n-1)}\left(t_{j+1}\right) & =x^{(2 n-1)}\left(t_{j}^{+}\right)-\int_{t_{j}}^{t_{j+1}} p(t) x^{r}(t-\tau) d t \\
& \leq x^{(2 n-1)}\left(t_{j}^{+}\right)-\left[x\left(t_{j}-\tau\right)\right]^{r} \int_{t_{j}}^{t_{j+1}} p(t) d t
\end{aligned}
$$




$$
=a_{j}^{(2 n-1)} x^{(2 n-1)}\left(t_{j}\right)-\left[x\left(t_{j}-\tau\right)\right]^{r} \int_{t_{j}}^{t_{j+1}} p(t) d t .
$$

Similarly to (11), we have

$$
\begin{aligned}
& x^{(2 n-1)}\left(t_{j+2}\right)=a_{j+1}^{(2 n-1)} x^{(2 n-1)}\left(t_{j+1}\right)-\left[x\left(t_{j+1}-\tau\right)\right]^{r} \int_{t_{j+1}}^{t_{j+2}} p(t) d t \\
& \leq a_{j+1}^{(2 n-1)}\left[a_{j}^{(2 n-1)} x^{(2 n-1)}\left(t_{j}\right)-\left[x\left(t_{j}-\tau\right)\right]^{r} \int_{t_{j}}^{t_{j+1}} p(t) d t\right]-\left[x\left(t_{j}-\tau\right)\right]^{r} \int_{t_{j+1}}^{t_{j+2}} p(t) d t \\
& \leq a_{j+1}^{(2 n-1)}\left\{a_{j}^{(2 n-1)} x^{(2 n-1)}\left(t_{j}\right)-\left[x\left(t_{j}-\tau\right)\right]^{r}\left[\int_{t_{j}}^{t_{j+1}} p(t) d t\right.\right. \\
& \left.\left.\quad+\frac{1}{a_{j+1}^{(2 n-1)}} \int_{t_{j+1}}^{t_{j+2}} p(t) d t\right]\right\} .
\end{aligned}
$$

By mathematical induction we have for any natural number $m \geq 2$,

$$
\begin{aligned}
& x^{(2 n-1)}\left(t_{j+m}\right) \leq a_{j+m-1}^{(2 n-1)} a_{j+m-2}^{(2 n-1)} \cdots a_{j+1}^{(2 n-1)}\left\{a_{j+1}^{(2 n-1)} x^{(2 n-1)}\left(t_{j}\right)\right. \\
&-\left[x\left(t_{j}-\tau\right)\right]^{r}\left[\int_{t_{j}}^{t_{j+1}} p(t) d t+\frac{1}{a_{j+1}^{(2 n-1)}} \int_{t_{j+1}}^{t_{j+2}} p(t) d t+\frac{1}{a_{j+2}^{(2 n-1)} a_{j+1}^{(2 n-1)}} \int_{t_{j+2}}^{t_{j+3}} p(t) d t\right. \\
&\left.\left.+\cdots+\frac{1}{a_{j+m-1}^{(2 n-1)} a_{j+m-2}^{(2 n-1)} \cdots a_{j+1}^{(2 n-1)}} \int_{t_{j+m-1}}^{t_{j+m}} p(t) d t\right]\right\} .
\end{aligned}
$$

By $(7),(13)$ and $a_{k}^{(i)}>0$, for all sufficiently large $m$, we have

$$
x^{(2 n-1)}\left(t_{j+m}\right)<0 .
$$

This contradicts the fact that $x^{(2 n-1)}(t)>0$ for all sufficiently large $t$. So every solution of (1) is oscillatory. The proof of Theorem 1 is completed.

Corollary 1. Assume that conditions $(A)$ and $(B)$ hold, and $a_{k}^{(0)} \geq 1$, $a_{k}^{(2 n-1)} \leq 1$ for $k=1,2, \ldots$ If $\int^{+\infty} p(t) d t=+\infty$, then every solution of (1) is oscillatory.

Proof. By $a_{k}^{(2 n-1)} \leq 1$, we have

$$
\frac{1}{a_{1}^{(2 n-1)} a_{2}^{(2 n-1)} \cdots a_{k}^{(2 n-1)}} \int_{t_{k}}^{t_{k+1}} p(t) d t \geq \int_{t_{k}}^{t_{k+1}} p(t) d t .
$$


As $m \rightarrow+\infty, \int_{t_{0}}^{t_{m+1}} p(t) d t \rightarrow+\infty$, condition (7) of Theorem 1 holds. By Theorem 1, we know that every solution of (1) is oscillatory.

Corollary 2. Assume that conditions $(A)$ and $(B)$ hold and there exists an $\alpha>0$ such that $a_{k}^{(0)} \geq 1, \frac{1}{a_{k}^{(2 n-1)}} \geq\left(\frac{t_{k+1}}{t_{k}}\right)^{\alpha}$ for $k=1,2, \ldots$. If $\int^{+\infty} t^{\alpha} p(t) d t=$ $+\infty$, then every solution of $(1)$ is oscillatory.

Proof. By $\frac{1}{a_{k}^{(2 n-1)}} \geq\left(\frac{t_{k+1}}{t_{k}}\right)^{\alpha}$, we have

$$
\frac{1}{a_{1}^{(2 n-1)} a_{2}^{(2 n-1)} \cdots a_{k}^{(2 n-1)}} \int_{t_{k}}^{t_{k+1}} p(t) d t \geq\left(\frac{1}{t_{1}}\right)^{\alpha} \int_{t_{k}}^{t_{k+1}} t^{\alpha} p(t) d t
$$

Then

$$
\int_{t_{0}}^{t_{1}} p(t) d t+\frac{1}{a_{1}^{(2 n-1)}} \int_{t_{1}}^{t_{2}} p(t) d t+\frac{1}{a_{1}^{(2 n-1)} a_{2}^{(2 n-1)}} \int_{t_{2}}^{t_{3}} p(t) d t \geq\left(\frac{1}{t_{1}}\right)^{\alpha} \int_{t_{1}}^{t_{m+1}} t^{\alpha} p(t) d t .
$$

As $m \rightarrow+\infty, \int_{t_{1}}^{t_{m+1}} p(t) d t \rightarrow+\infty$, condition (7) of Theorem 1 holds. Theorem 1 implies that every solution of (1) is oscillatory.

Theorem 2. Suppose that conditions $(A),(B)$ hold and for any natural number $k, t_{k}-t_{k-1}>\tau>0$. Let either

$$
\begin{aligned}
(a): & \int_{t_{0}+\tau}^{t_{1}} p(t) d t+\frac{\left[a_{1}^{(0)}\right]^{r}}{a_{1}^{(2 n-1)}} \int_{t_{1}+\tau}^{t_{2}} p(t) d t+\frac{\left[a_{1}^{(0)}\right]^{r}\left[a_{2}^{(0)}\right]^{r}}{a_{1}^{(2 n-1)} a_{2}^{(2 n-1)}} \int_{t_{2}+\tau}^{t_{3}} p(t) d t \\
& +\cdots+\frac{\left[a_{1}^{(0)}\right]^{r}\left[a_{2}^{(0)}\right]^{r} \cdots\left[a_{m}^{(0)}\right]^{r}}{a_{1}^{(2 n-1)} a_{2}^{(2 n-1)} \cdots a_{m}^{(2 n-1)}} \int_{t_{m}+\tau}^{t_{m+1}} p(t) d t+\cdots=+\infty
\end{aligned}
$$

or

$$
\begin{aligned}
(b): & \int_{t_{0}}^{t_{0}+\tau} p(t) d t+\frac{1}{a_{1}^{(2 n-1)}} \int_{t_{1}}^{t_{1}+\tau} p(t) d t+\frac{\left[a_{1}^{(0)}\right]^{r}}{a_{1}^{(2 n-1)} a_{2}^{(2 n-1)}} \int_{t_{2}}^{t_{2}+\tau} p(t) d t \\
& +\cdots+\frac{\left[a_{1}^{(0)}\right]^{r}\left[a_{2}^{(0)}\right]^{r} \cdots\left[a_{m-1}^{(0)}\right]^{r}}{a_{1}^{(2 n-1)} a_{2}^{(2 n-1)} \cdots a_{m}^{(2 n-1)}} \int_{t_{m}}^{t_{m}+\tau} p(t) d t+\cdots=+\infty
\end{aligned}
$$

hold. Then every solution of (1) is oscillatory.

Proof. Suppose that (1) has a non-oscillatory solution $x(t)$. Without the loss of generality suppose that $T^{\prime}=t_{0}$ and $x(t)>0\left(t \geq t_{0}\right)$. Lemma 3 and (1) imply that there exists $T^{\prime}=t_{0}$ such that for $t \geq T^{\prime}$,

$$
x^{(2 n)}(t) \leq 0, \quad x^{(2 n-1)}(t)>0, \quad x^{\prime}(t)>0, \quad x(t)>0 .
$$


Then

$$
\begin{aligned}
x^{(2 n-1)}\left(t_{k+m}\right) & \leq x^{(2 n-1)}\left(t_{k+m-1}^{+}\right)=a_{k+m-1}^{(2 n-1)} x^{(2 n-1)}\left(t_{k+m-1}\right) \\
& \leq a_{k+m-1}^{(2 n-1)} x^{(2 n-1)}\left(t_{k+m-2}^{+}\right) \\
& =a_{k+m-1}^{(2 n-1)} a_{k+m-2}^{(2 n-1)} x^{(2 n-1)}\left(t_{k+m-2}\right) \\
& \leq \cdots \cdots \cdots \cdots \\
& \leq a_{k+m-1}^{(2 n-1)} a_{k+m-2}^{(2 n-1)} \cdots a_{3}^{(2 n-1)} a_{2}^{(2 n-1)} a_{1}^{(2 n-1)} x^{(2 n-1)}\left(t_{1}\right), \\
x\left(t_{k}^{+}\right) & =a_{k}^{(0)} x\left(t_{k}\right) \geq a_{k}^{(0)} x\left(t_{k-1}^{+}\right) \geq a_{k}^{(0)} a_{k-1}^{(0)} x\left(t_{k-1}\right) \\
& \geq \cdots \cdots \cdots \cdots \\
& \geq a_{k}^{(0)} a_{k-1}^{(0)} \cdots a_{2}^{(0)} a_{1}^{(0)} x\left(t_{1}\right) .
\end{aligned}
$$

If condition $(a)$ holds, then for $t \in\left(t_{1}, t_{2}\right]$,

$$
x^{(2 n)}(t)=-p(t)[x(t-\tau)]^{r} .
$$

Integrating (17) from $t_{1}$ to $t_{2}$, we have

$$
\begin{aligned}
x^{(2 n-1)}\left(t_{2}\right) & =x^{(2 n-1)}\left(t_{1}^{+}\right)-\int_{t_{1}}^{t_{2}} p(t)[x(t-\tau)]^{r} d t \\
& \leq a_{1}^{(2 n-1)} x^{(2 n-1)}\left(t_{1}\right)-\left[x\left(t_{1}^{+}\right)\right]^{r} \int_{t_{1}+\tau}^{t_{2}} p(t) d t \\
& \leq a_{1}^{(2 n-1)} x^{(2 n-1)}\left(t_{1}\right)-\left[a_{1}^{(0)}\right]^{r}\left[x\left(t_{1}\right)\right]^{r} \int_{t_{1}+\tau}^{t_{2}} p(t) d t .
\end{aligned}
$$

Similarly,

$$
\begin{aligned}
x^{(2 n-1)}\left(t_{3}\right) \leq & a_{2}^{(2 n-1)} x^{(2 n-1)}\left(t_{2}\right)-\left[x\left(t_{2}^{+}\right)\right]^{r} \int_{t_{2}+\tau}^{t_{3}} p(t) d t \\
\leq & a_{2}^{(2 n-1)}\left[a_{1}^{(2 n-1)} x^{(2 n-1)}\left(t_{1}\right)-\left[a_{1}^{(0)}\right]^{r}\left[x\left(t_{1}\right)\right]^{r} \int_{t_{1}+\tau}^{t_{2}} p(t) d t\right] \\
& -\left[a_{1}^{(0)}\right]^{r}\left[a_{2}^{(0)}\right]^{r}\left[x\left(t_{1}\right)\right]^{r} \int_{t_{2}+\tau}^{t_{3}} p(t) d t \\
= & a_{2}^{(2 n-1)} a_{1}^{(2 n-1)} x^{(2 n-1)}\left(t_{1}\right) \\
& -\left[x\left(t_{1}\right)\right]^{r}\left[\left[a_{1}^{(0)}\right]^{r} a_{2}^{(2 n-1)} \int_{t_{1}+\tau}^{t_{2}} p(t) d t+\left[a_{1}^{(0)}\right]^{r}\left[a_{2}^{(0)}\right]^{r} \int_{t_{2}+\tau}^{t_{3}} p(t) d t\right] .
\end{aligned}
$$


Suppose that, for any natural number $k$

$$
\begin{aligned}
x^{(2 n-1)}\left(t_{k}\right) \leq & a_{1}^{(2 n-1)} a_{2}^{(2 n-1)} \cdots a_{k-2}^{(2 n-1)} a_{k-1}^{(2 n-1)} x^{(2 n-1)}\left(t_{1}\right) \\
& -\left[x\left(t_{1}\right)\right]^{r}\left\{\sum_{i=1}^{k-1}\left[a_{1}^{(0)}\right]^{r} \cdots\left[a_{i}^{(0)}\right]^{r} a_{i+1}^{(2 n-1)} \cdots a_{k-1}^{(2 n-1)} \int_{t_{i}+\tau}^{t_{i+1}} p(t) d t\right\} .
\end{aligned}
$$

Integrating (17) from $t_{k}$ to $t_{k+1}$, we have

$$
\begin{aligned}
x^{(2 n-1)}\left(t_{k+1}\right) \leq & a_{k}^{(2 n-1)} x^{(2 n-1)}\left(t_{k}\right)-\left[x\left(t_{k}^{+}\right)\right]^{r} \int_{t_{k}+\tau}^{t_{k+1}} p(t) d t \\
\leq & a_{k}^{(2 n-1)} a_{1}^{(2 n-1)} a_{2}^{(2 n-1)} \cdots a_{k-1}^{(2 n-1)} x^{(2 n-1)}\left(t_{1}\right) \\
& -\left[x\left(t_{1}\right)\right]^{r}\left\{\sum_{i=1}^{k-1}\left[a_{1}^{(0)}\right]^{r} \cdots\left[a_{i}^{(0)}\right]^{r} a_{i+1}^{(2 n-1)} \cdots a_{k-1}^{(2 n-1)} \int_{t_{i}+\tau}^{t_{i+1}} p(t) d t\right\} \\
& -\left[a_{1}^{(0)}\right]^{r}\left[a_{2}^{(0)}\right]^{r} \cdots\left[a_{k-1}^{(0)}\right]^{r}\left[a_{k}^{(0)}\right]^{r}\left[x\left(t_{1}\right)\right]^{r} \int_{t_{k}+\tau}^{t_{k+1}} p(t) d t \\
= & a_{1}^{(2 n-1)} a_{2}^{(2 n-1)} \cdots a_{k-1}^{(2 n-1)} a_{k}^{(2 n-1)} x^{(2 n-1)}\left(t_{1}\right) \\
& -\left[x\left(t_{1}\right)\right]^{r}\left\{\sum_{i=1}^{k}\left[a_{1}^{(0)}\right]^{r} \cdots\left[a_{i}^{(0)}\right]^{r} a_{i+1}^{(2 n-1)} \cdots a_{k}^{(2 n-1)} \int_{t_{i}+\tau}^{t_{i+1}} p(t) d t\right\} .
\end{aligned}
$$

By mathematical induction, we know that for any natural number $m$

$$
\begin{aligned}
x^{(2 n-1)}\left(t_{m}\right) \leq & a_{1}^{(2 n-1)} a_{2}^{(2 n-1)} \cdots a_{m-1}^{(2 n-1)} x^{(2 n-1)}\left(t_{1}\right) \\
& -\left[x\left(t_{1}\right)\right]^{r}\left\{\sum_{i=1}^{m-1}\left[a_{1}^{(0)}\right]^{r} \cdots\left[a_{i}^{(0)}\right]^{r} a_{i+1}^{(2 n-1)} \cdots a_{m-1}^{(2 n-1)} \int_{t_{i}+\tau}^{t_{i+1}} p(t) d t\right\} .
\end{aligned}
$$

Therefore

$$
\begin{aligned}
x^{(2 n-1)}\left(t_{m}\right) \leq & a_{1}^{(2 n-1)} a_{2}^{(2 n-1)} \cdots a_{m-1}^{(2 n-1)}\left[x\left(t_{1}\right)\right]^{r}\left\{\frac{x^{(2 n-1)}\left(t_{1}\right)}{\left[x\left(t_{1}\right)\right]^{r}}\right. \\
& \left.-\left\{\sum_{i=1}^{m-1} \frac{\left[a_{1}^{(0)}\right]^{r} \cdots\left[a_{i}^{(0)}\right]^{r}}{a_{1}^{(2 n-1)} \cdots a_{i}^{(2 n-1)}} \int_{t_{i}+\tau}^{t_{i+1}} p(t) d t\right\}\right\} .
\end{aligned}
$$

The above inequality and (14) imply that $x^{(2 n-1)}\left(t_{m}\right) \leq 0$ for $m$ large enough. Thus $x^{(2 n-1)}(t) \leq 0$ for all sufficiently large $t$. This contradicts $x^{(2 n-1)}(t)>0$ for $t \geq T^{\prime}$. So every solution of (1) is oscillatory. 
If condition $(b)$ holds, integrating (17) from $t_{1}$ to $t_{2}$, we have

$$
\begin{aligned}
x^{(2 n-1)}\left(t_{2}\right) & =x^{(2 n-1)}\left(t_{1}^{+}\right)-\int_{t_{1}}^{t_{2}} p(t)[x(t-\tau)]^{r} d t \\
& \leq x^{(2 n-1)}\left(t_{1}^{+}\right)-\int_{t_{1}}^{t_{1}+\tau} p(t)[x(t-\tau)]^{r} d t \\
& \leq a_{1}^{(2 n-1)} x^{(2 n-1)}\left(t_{1}\right)-\left[x\left(t_{1}-\tau\right)\right]^{r} \int_{t_{1}}^{t_{1}+\tau} p(t) d t .
\end{aligned}
$$

Similarly,

$$
\begin{aligned}
x^{(2 n-1)}\left(t_{3}\right) \leq & a_{2}^{(2 n-1)} x^{(2 n-1)}\left(t_{2}\right)-\left[x\left(t_{2}-\tau\right)\right]^{r} \int_{t_{2}}^{t_{2}+\tau} p(t) d t \\
\leq & a_{2}^{(2 n-1)} x^{(2 n-1)}\left(t_{2}\right)-\left[x\left(t_{1}^{+}\right)\right]^{r} \int_{t_{2}}^{t_{2}+\tau} p(t) d t \\
\leq & a_{2}^{(2 n-1)}\left\{a_{1}^{(2 n-1)} x^{(2 n-1)}\left(t_{1}\right)-\left[x\left(t_{1}-\tau\right)\right]^{r} \int_{t_{1}}^{t_{1}+\tau} p(t) d t\right\} \\
& -\left[a_{1}^{(0)}\right]^{r}\left[x\left(t_{1}-\tau\right)\right]^{r} \int_{t_{2}}^{t_{2}+\tau} p(t) d t \\
= & a_{2}^{(2 n-1)} a_{1}^{(2 n-1)} x^{(2 n-1)}\left(t_{1}\right) \\
& -\left[x\left(t_{1}-\tau\right)\right]^{r}\left\{a_{2}^{(2 n-1)} \int_{t_{1}}^{t_{1}+\tau} p(t) d t+\left[a_{1}^{(0)}\right]^{r} \int_{t_{2}}^{t_{2}+\tau} p(t) d t\right\} .
\end{aligned}
$$

By mathematical induction, for any natural number $m$

$$
\begin{aligned}
x^{(2 n-1)}\left(t_{m}\right) \leq & a_{1}^{(2 n-1)} a_{2}^{(2 n-1)} \cdots a_{m-1}^{(2 n-1)} x^{(2 n-1)}\left(t_{1}\right) \\
& -\left[x\left(t_{1}-\tau\right)\right]^{r}\left\{\sum_{i=1}^{m-1}\left[a_{1}^{(0)}\right]^{r} \cdots\left[a_{i-1}^{(0)}\right]^{r} a_{i+1}^{(2 n-1)} \cdots a_{m-1}^{(2 n-1)} \int_{t_{i}}^{t_{i}+\tau} p(t) d t\right\} .
\end{aligned}
$$

Hence

$$
x^{(2 n-1)}\left(t_{m}\right) \leq a_{1}^{(2 n-1)} a_{2}^{(2 n-1)} \cdots a_{m-1}^{(2 n-1)}\left[x\left(t_{1}-\tau\right)\right]^{r}\left\{\frac{x^{(2 n-1)}\left(t_{1}\right)}{\left[x\left(t_{1}-\tau\right)\right]^{r}}\right.
$$




$$
\left.-\left\{\sum_{i=1}^{m-1} \frac{\left[a_{1}^{(0)}\right]^{r} \cdots\left[a_{i-1}^{(0)}\right]^{r}}{a_{1}^{(2 n-1)} \cdots a_{i}^{(2 n-1)}} \int_{t_{i}}^{t_{i}+\tau} p(t) d t\right\}\right\} .
$$

The above inequality and (15) imply that $x^{(2 n-1)}\left(t_{m}\right) \leq 0$ for $m$ large enough. So $x^{(2 n-1)}(t) \leq 0$ for all sufficiently large $t$. This contradicts $x^{(2 n-1)}(t)>0$ for $t \geq T^{\prime}$. So every solution of (1) is oscillatory.

Summing up the above discussion, we can see that every solution of (1) is oscillatory. The proof is completed.

Consider

$$
\left\{\begin{array}{l}
x^{(2 n)}(t)+p(t)|x(t-\tau)|^{r} \operatorname{sgn}(x(t-\tau))=0, \quad t \geq t_{0} \\
x(t)=\phi(t), \quad t_{0}-\tau \leq t \leq t_{0}
\end{array}\right.
$$

and

$$
\left\{\begin{array}{l}
x^{(2 n)}(t)+p(t)|x(t-\tau)|^{r} \operatorname{sgn}(x(t-\tau))=0, \\
x^{(i)}\left(t_{k}^{+}\right)=I_{k(i)}\left(x^{(i)}\left(t_{k}\right)\right) \quad \text { for } \quad i=0,1, \ldots, 2 n-1, \\
x^{(i)}\left(t_{0}^{+}\right)=x_{0}^{(i)} \text { for } \quad k=0,1, \ldots, \\
x(t)=\phi(t), \quad t_{0}-\tau \leq t \leq t_{0} .
\end{array}\right.
$$

where $I_{k(i)}(x)$ is continuous on $(-\infty,+\infty)$ and $x I_{k(i)}(x)>0(x \neq 0), k=$ $1,2, \ldots, \varphi(t):\left[t_{0}-\tau, t_{0}\right] \rightarrow R$ has at most a finite number of discontinuous points of first kind and is left continuous at those points.

Theorem 3. If $p(t)>0$ is continuous on $[0,+\infty), r>0, r \neq 1$, then for any $\left\{t_{m}\right\}: 0<t_{1}<t_{2}<\cdots<t_{m}<\cdots, t_{m}-t_{m-1}>\tau>0$, one can find suitable impulsive functions $I_{k(i)}(x)$ such that under the impulsive effects (18) transforms to (19), all solutions of (19) are oscillatory.

Proof. Let $c_{k}=\int_{t_{k}+\tau}^{t_{k+1}} p(t) d t, c_{0}=1$ and $I_{k(i)}(x)=d_{k} x\left(a_{k}^{(i)}=d_{k}\right)$, where $d_{k}=\left(\frac{c_{k-1}}{c_{k}}\right)^{\frac{1}{r-1}}, k=1,2, \ldots$ Then

$$
d_{1}^{r-1}=\frac{1}{c_{1}}, \quad\left(d_{1} d_{2}\right)^{r-1}=\frac{1}{c_{2}}, \ldots,\left(d_{1} d_{2} \cdots d_{m}\right)^{r-1}=\frac{1}{c_{m}} .
$$

Hence

$$
\begin{gathered}
\int_{t_{0}+\tau}^{t_{1}} p(t) d t+\frac{\left[a_{1}^{(0)}\right]^{r}}{a_{1}^{(2 n-1)}} \int_{t_{1}+\tau}^{t_{2}} p(t) d t+\frac{\left[a_{1}^{(0)}\right]^{r}\left[a_{2}^{(0)}\right]^{r}}{a_{1}^{(2 n-1)} a_{2}^{(2 n-1)}} \int_{t_{2}+\tau}^{t_{3}} p(t) d t \\
+\cdots+\frac{\left[a_{1}^{(0)}\right]^{r}\left[a_{2}^{(0)}\right]^{r} \cdots\left[a_{m}^{(0)}\right]^{r}}{a_{1}^{(2 n-1)} a_{2}^{(2 n-1)} \cdots a_{m}^{(2 n-1)}} \int_{t_{m}+\tau}^{t_{m+1}} p(t) d t \\
\geq d_{1}^{r-1} c_{1}+\left(d_{1} d_{2}\right)^{r-1} c_{2}+\cdots+\left(d_{1} d_{2} \cdots d_{m}\right)^{r-1} c_{m}=m .
\end{gathered}
$$

Therefore the condition (a) of Theorem 2 holds. We can see that every solution of (19) is oscillatory. 
Remark 3. Though the condition on $p(t)$ cannot guarantee that all solutions of (18) are oscillatory, we can see from Theorem 3 that if we give some suitable impulsive effects to it, all solutions can become oscillatory.

\section{EXAMPLES}

Example 1. Consider the system

$$
\left\{\begin{array}{l}
x^{(2 n)}(t)+x^{2 n-1}\left(t-\frac{1}{2}\right)=0, \quad t \geq t_{0}=1, \quad t \neq 2^{k}, \quad k=1,2, \ldots \\
x\left(\left(2^{k}\right)^{+}\right)=2 x\left(2^{k}\right), \quad x^{(i)}\left(\left(2^{k}\right)^{+}\right)=\frac{k}{k+1} x^{(i)}\left(2^{k}\right), \quad i=1, \ldots, 2 n-1, \\
x(1)=x_{0}, \quad x^{(i)}\left(1^{+}\right)=x_{0}^{(i)} \\
x(t)=\phi(t), \quad t \in\left[\frac{1}{2}, 1\right] .
\end{array}\right.
$$

where $a_{k}^{(0)}=2>1, a_{k}^{(i)}=\frac{k}{k+1}, i=1,2, \ldots, 2 n-1, p(t)=1, t_{k}=2^{k}, \tau=\frac{1}{2}>0$, $t_{k+1}-t_{k}=2^{k+1}-2^{k}=2^{k}>\frac{1}{2}, t_{0}=1, \gamma=2 n-1$. It is obvious that condition $(A)$ is satisfied. As to condition $(B)$, for $i>1, a_{k}^{(i)}=\frac{k}{k+1}$ we have $\left(t_{1}-t_{0}\right)+\left(t_{2}-t_{1}\right)+\left(t_{3}-t_{2}\right)+\cdots+\left(t_{m+1}-t_{m}\right)+\cdots=1+2^{1}+2^{2}+\cdots+2^{m}+\cdots=+\infty$.

For $i=1, a_{k}^{(0)}=2, a_{k}^{(1)}=\frac{k}{k+1}$ we have

$$
\begin{aligned}
\left(t_{1}-t_{0}\right) & +\frac{1}{2 \times 2}\left(t_{2}-t_{1}\right)+\frac{1}{3 \times 2^{2}}\left(t_{3}-t_{2}\right)+\cdots+\frac{1}{(m+1) \times 2^{m}}\left(t_{m+1}-t_{m}\right)+\cdots \\
& =1+\frac{1}{2 \times 2} 2+\frac{1}{3 \times 2^{2}} 2^{2}+\cdots+\frac{1}{(m+1) \times 2^{m}} 2^{m}+\cdots \\
& =1+\frac{1}{2}+\frac{1}{3}+\cdots+\frac{1}{m+1}+\cdots=+\infty
\end{aligned}
$$

So, condition $(B)$ holds.

Since $a_{k}^{(2 n-1)}=\frac{k}{k+1}$, we have

$$
\begin{aligned}
& \int_{t_{0}}^{t_{1}} p(t) d t+\frac{1}{a_{1}^{(2 n-1)}} \int_{t_{1}}^{t_{2}} p(t) d t+\frac{1}{a_{1}^{(2 n-1)} a_{2}^{(2 n-1)}} \int_{t_{2}}^{t_{3}} p(t) d t \\
& \quad+\cdots+\frac{1}{a_{1}^{(2 n-1)} a_{2}^{(2 n-1)} \cdots a_{m}^{(2 n-1)}} \int_{t_{m}}^{t_{m+1}} p(t) d t+\cdots \\
& \quad \int_{t_{0}}^{t_{1}} 1 d t+2 \int_{t_{1}}^{t_{2}} 1 d t+3 \int_{t_{2}}^{t_{3}} 1 d t+\cdots+(m+1) \int_{t_{m}}^{t_{m+1}} 1 d t+\cdots \\
& =\left(t_{1}-t_{0}\right)+2\left(t_{2}-t_{1}\right)+3\left(t_{3}-t_{2}\right)+\cdots+(m+1)\left(t_{m+1}-t_{m}\right)+\cdots \\
& =1+2 \times 2+3 \times 2^{2}+\cdots+(m+1) \times 2^{m}+\cdots=+\infty .
\end{aligned}
$$

Therefore we get that the conditions of Theorem 1 hold. So we can see that every solution of $(20)$ defined on $\left[t_{0},+\infty\right)$ is oscillatory. 
Example 2. Consider the sublinear system:

$$
\left\{\begin{array}{l}
x^{(2 n)}(t)+\frac{2}{t} x^{\frac{1}{9}}(t-\ln 2)=0, \quad t \geq t_{0}=\frac{1}{2}, \quad t \neq k, \quad k=1,2, \ldots \\
x\left(k^{+}\right)=x(k), \quad x^{(i)}\left(k^{+}\right)=\frac{k}{k+1} x^{(i)}(k), \quad i=1, \ldots, 2 n-1, \\
x\left(\frac{1}{2}\right)=x_{0}, \quad x^{(i)}\left(\frac{1}{2}^{+}\right)=x_{0}^{(i)} \\
x(t)=\phi(t), \quad t \in\left[\frac{1}{2}-\ln 2, \frac{1}{2}\right]
\end{array}\right.
$$

where $a_{k}^{(0)}=1, a_{k}^{(i)}=\frac{k}{k+1}, i=1,2, \ldots, 2 n-1, \tau=\ln 2, t_{k}=k, t_{k+1}-t_{k}=1>$ $\ln 2, p(t)=\frac{2}{t}, \gamma=\frac{1}{9}, t_{0}=\frac{1}{2}$. It is obvious that condition $(A)$ is satisfied. As to condition $(B)$, for $i>1, a_{k}^{(i)}=\frac{k}{k+1}$ we have

$$
\left(t_{1}-t_{0}\right)+\left(t_{2}-t_{1}\right)+\left(t_{3}-t_{2}\right)+\cdots+\left(t_{m+1}-t_{m}\right)+\cdots=+\infty .
$$

For $i=1, a_{k}^{(0)}=1, a_{k}^{(1)}=\frac{k}{k+1}$ we obtain

$$
\begin{aligned}
\left(t_{1}-t_{0}\right)+\frac{1}{2}\left(t_{2}-t_{1}\right)+\frac{1}{3}\left(t_{3}-t_{2}\right) & +\cdots+\frac{1}{m+1}\left(t_{m+1}-t_{m}\right)+\cdots \\
& =\frac{1}{2}+\frac{1}{2}+\frac{1}{3}+\cdots+\frac{1}{m+1}+\cdots=+\infty .
\end{aligned}
$$

So, condition $(B)$ holds.

Let $\alpha=1, a_{k}^{(0)}=1, \frac{1}{a_{k}^{(2 n-1)}}=\frac{k+1}{k} \geq\left(\frac{t_{k+1}}{t_{k}}\right)^{\alpha}=\frac{k+1}{k}, \int^{+\infty} t \cdot p(t) d t=+\infty$. Therefore the conditions of Corollary 2 are satisfied. Then every solution of (21) defined on $\left[t_{0},+\infty\right)$ is oscillatory.

\section{ACKNOWLEDGEMENT}

This work was partially supported by Natural Science Foundation of Guangdong (011471) and Natural Science Foundation of Guangdong Higher Education (0120), Science and Technology Plan Project of Guangzhou(2006J1-C0341).

\section{REFERENCES}

1. K. Gopalsamy and B. G. Zhang, On delay differential equations with impulses. $J$. Math. Anal. Appl. 139(1989), No. 1, 110-122.

2. D. D. Bainov, M. B. Dimitrova, and A. B. Dishliev, Oscillating solutions of nonlinear impulsive differential equations with a deviating argument. Note Mat. 15(1995), No. 1, 45-54 (1997).

3. L. Berezansky and E. Braverman, Oscillation of a linear delay impulsive differential equation. Comm. Appl. Nonlinear Anal. 3(1996), No. 1, 61-77.

4. Y. Zhang, A. ZhaO, and J. Yan, Oscillation criteria for impulsive delay differential equations. J. Math. Anal. Appl. 205(1997), No. 2, 461-470.

5. D. D. Bainov, Yu. I. Domshlak, and P. S. Simeonov, On the oscillation properties of first-order impulsive differential equations with a deviating argument. Israel J. Math. 98(1997), 167-187.

6. D. D. Bainov, Yu. I. Domashlak, and P. S. Simeonov, Sturmian comparison theory for impulsive differential inequalities and equations. Arch. Math. (Basel) 67(1996), No. $1,35-49$. 
7. Yongshao Chen and Weizhen Feng, Oscillations of second order nonlinear ODE with impulses. J. Math. Anal. Appl. 210(1997), No. 1, 150-169.

8. Chunchao Huang, Oscillation and nonoscillation for second order linear impulsive differential equations. J. Math. Anal. Appl. 214(1997), No. 2, 378-394.

9. D. Bainov and M. Dimitrova, Oscillation of sub- and superlinear impulsive differential equations with constant delay. Appl. Anal. 64(1997), No. 1-2, 57-67.

10. L. Berezansky and E. Braverman, On oscillation of a second order impulsive linear delay differential equation. J. Math. Anal. Appl. 233(1999), No. 1, 276-300.

11. Weizhen Feng and Yongshao Chen, Oscillations of second order functional differential equations with impulses. Advances in impulsive differential equations. Dyn. Contin. Discrete Impuls. Syst. Ser. A Math. Anal. 9(2002), No. 3, 367-376.

12. Yongchao Chen and Weizhen Feng, Oscillations of higher-order linear ODEs with impulses. (Chinese) J. South China Normal Univ. Natur. Sci. Ed. 2003, No. 3, 14-19.

13. Weizhen Feng, Oscillations of fourth order ODE with impulses. Ann. Differential Equations 19(2003), No. 2, 136-145.

14. L. Wen and Y. Chen, Razumikhin type theorems for functional differential equations with impulses. Dynam. Contin. Discrete Impuls. Systems 6(1999), No. 3, 389-400.

15. J. Shen, Z. LuO, and X. Liu, Impulsive stabilization of functional-differential equations via Liapunov functionals. J. Math. Anal. Appl. 240(1999), No. 1, 1-15.

(Received 6.02.2006)

Authors' addresses:

Chaolong Zhang Department of Computation Science

Zhongkai University of Agriculture and Technology

Guangzhou, 510225

P.R. China

E-mail: zhcl88@126.com

Weizhen Feng

Department of Mathematics

South China Normal University

Guangzhou, 510631

P.R. China 